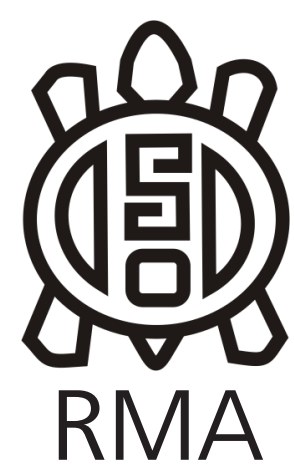

Dossier

\section{Historias de agua y fuego. Modificaciones del material lítico en sitios del bosque norpatagónico}

\author{
Stories of water and fire. Modification of lithic assemblages from forest \\ northpatagonic archaeological sites
}

* Mariana Carballido Calatayud y ** Cristina Bellelli

* CONICET, Instituto Nacional de Antropología y Pensamiento Latinoamericano (INAPL), Universidad de Buenos Aires, Argentina. E-mail: mcarballidocalata@hotmail.com ** CONICET, Instituto Nacional de Antropología y Pensamiento Latinoamericano (INAPL).

E-mail: crisbellelli@yahoo.com.ar

\title{
Resumen
}

Entender los aspectos generales del bosque, especialmente la dinámica de las perturbaciones naturales (incendios) y la elevada humedad, así como los propios de los sitios, tales como su ubicación topográfica, las dinámicas de erosión / depositación y los vinculados con la ocupación humana, nos permitieron caracterizar a los procesos de formación del registro arqueológico que afectan recurrentemente a los conjuntos líticos de este ambiente.

El análisis de tres casos ubicados en localizaciones diferentes del área boscosa comprendida entre el límite sur del Parque Nacional Nahuel Huapi y el límite norte del Parque Nacional Los Alerces permitieron determinar que el fuego, sobre todo el producido por los incendios forestales, es un poderoso agente transformador de los conjuntos líticos confeccionados en diferentes materias primas, pero sobre todo de aquellas con alto contenido de sílice. La humedad de los depósitos actuó de manera heterogénea dependiendo de la localización de los sitios, siendo sus efectos los propios de flujos de baja energía (películas de sedimento o manchas blancas).

Palabras clave: Bosque; procesos de formación; fuego; humedad; conjuntos líticos.

\begin{abstract}
Understanding the general aspects of the forest environment, especially the dynamics of natural disturbances (fires) and high humidity, as well as those of the sites, such as their topographic location, erosion / deposition dynamics and those related to human occupation, allowed us to characterize the formation processes of the archaeological record that recurrently affect lithic assemblages of this environment.

The analysis of three cases located in different locations of the forest area between the southern limit of the Nahuel Huapi National Park and the northern limit of the Los Alerces National Park made it possible to determine that fire, especially that caused by forest fires, is a powerful transformer agent of lithic assemblages of different raw materials, but especially those with high silica content. The humidity of the deposits acted heterogeneously depending on the location of the sites, their effects being those of low energy flows (sediment films or white spots).
\end{abstract}

Keywords: Forest; formation processes; fire; humidity; lithic assemblages.

El escenario donde se desarrolla nuestra investigación es la región cordillerana comprendida entre el límite sur del Parque Nacional Nahuel Huapi y el límite norte del Parque Nacional Los Alerces. Caracterizada por el desarrollo de un bosque mixto de Nothofagus sp. y Austrocedrus chilensis (ciprés), asociados a radal (Lomatia hirsuta), maitén (Maytenus boaria), chacay (Discaria trinervis), etc. y una variedad muy importante de especies no arbóreas, que en algunos sectores forma un denso sotobosque. Se desarrolla en un paisaje glaciario de lagos y valles fluviales diseñados por la orogenia andina.

Las excavaciones realizadas en tres de los sitios estratificados ubicados en paredones con arte rupestre brindaron los conjuntos líticos cuyas historias de vida son objeto de este trabajo. De norte a sur son, Paredón Lanfré (PL), en el valle del río Manso inferior (Río Negro), Risco de Azócar 1 (RA1) en el valle del río Epuyén (Chubut) y Cerro Pintado (CP), en cercanías de la localidad Cholila (Chubut) cuyas ocupaciones se remontan a los últimos 1500 años (Figura 1).

El sitio PL es un paredón que forma parte de la barda que enmarca la planicie de inundación del río Manso inferior a unos $500 \mathrm{~m}$ sobre el nivel del mar y a unos $1000 \mathrm{~m}$ de la margen sur del río Manso (Bellelli et al. 
2007; Fernández et al. 2013). Durante las excavaciones se obtuvieron muestras de carbón y se detectaron dos fogones contiguos en la base de la excavación. También se identificaron algunas inversiones en los fechados que, sumadas a la dispersión vertical de materiales arqueológicos y modernos podrían deberse

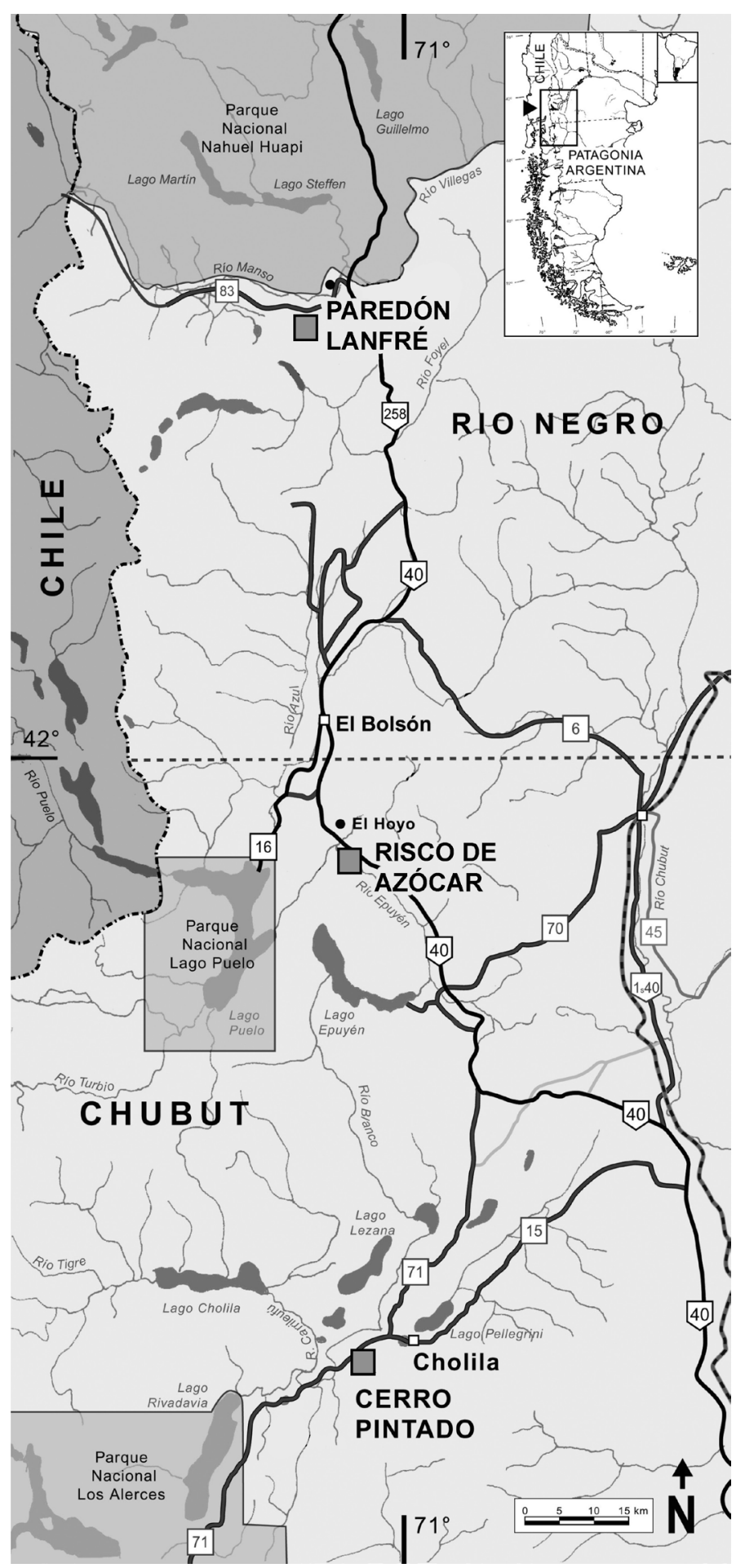

a la acción erosiva de escurrimientos de agua durante el invierno (Carballido Calatayud et al. 2016). El sitio RA1 se ubica en la planicie de inundación del río Epuyén, sobre la ladera del Cerro Pirque, a unos $200 \mathrm{~m}$ del río en un terreno plano y con escasa pendiente. Durante las excavaciones no se identificaron estructuras de combustión aunque sí se observaron pequeñas concentraciones de carbón vegetal de las que se tomaron muestras para fechar (Podestá et al. 2007). En los dos casos reseñados, al estar ubicados en la planicie de inundación de los ríos Manso inferior y Epuyén, vemos que prevalece el aporte sedimentario de origen fluvial y eólico y que se registran inundaciones que cubren los depósitos arqueológicos. Así, en PL y RA1 los materiales arqueológicos se disponen en una matriz franco arenosa que alcanza alrededor de $1,30 \mathrm{~m}$ en PL y $95 \mathrm{~cm}$ en RA1. Los fechados obtenidos en ambos depósitos indican que su formación abarca lapsos de algo más de 1500 años.

Finalmente, $\mathrm{CP}$ es una roca aborregada ubicada en una pendiente, a unos 100 metros por sobre la planicie de inundación de los ríos Blanco/ Carrileufú. En la excavación se identificaron dos estructuras de combustión claras, un fogón de cubeta y uno plano. En este sitio, a diferencia de lo que sucede en los anteriormente reseñados, predominan los procesos de remoción por sobre los de depositación. Tan es así que los depósitos tienen una potencia máxima de $30 \mathrm{~cm}$ y su rango cronológico comprende cerca de 1900 años (Bellelli et al. 2003).

La información referida a las diferencias en las tasas de depositación de cada caso muestra que la dinámica de sedimentación/erosión está fuertemente vinculada al emplazamiento de los sitios y es importante para valorar la magnitud y el impacto de los distintos agentes culturales y/o naturales formadores del registro.

\section{Fuego y agua en el bosque norpatagónico}

\section{El fuego}

Los incendios forestales constituyen una de las principales perturbaciones naturales, forman parte de la dinámica de este ambiente y brindan oportunidades para la renovación de los bosques maduros o crean condiciones para la colonización de espacios disponibles (Fuentes Quezada 1994; Kitzberger y Veblen 1999, entre otros).
Figura 1: Ubicación de los sitios Paredón Lanfré, Risco de Azócar 1 y Cerro Pintado.

Figure 1: Location of Paredón Lanfré, Risco de Azócar 1 and Cerro Pintado sites.
Diversos estudios paleoambientales documentan la existencia de incendios durante todo el Holoceno. En el sector norte del área de investigación para los últimos 1500 años, los testigos sedimentarios provenientes 
del Laguito del Morro muestran un pico de eventos de fuego tanto en frecuencia como en intensidad en el valle del río Manso (Giaché y Bianchi 2018). Por su parte, las muestras analizadas del Lago Mosquito y Laguna el Cóndor (Cholila), ubicadas ambas en el sector sur, mostraron que en los últimos dos milenios hubo un aumento en la humedad y un régimen de incendios fluctuante, muy frecuentes y poco severos, con cortos períodos libres de fuego (Iglesias et al. 2012).

La presencia de carbones y cenizas en los depósitos arqueológicos puede señalar tanto la acción de los incendios forestales como la actividad humana. Por ello registrar la presencia, extensión y definición clara de fogones es importante para entender el origen de las modificaciones en el material arqueológico. Así, se registraron fogones formatizados en $\mathrm{PL}$ y $\mathrm{CP}$ mientras que en los tres sitios se identificaron lentes carbonosas y carbones sueltos que pueden ser interpretados tanto como producto de la actividad humana como una señal de antiguos incendios forestales. Además, la información provista por los pobladores indica que en los últimos 50 años hubo numerosos episodios de incendios que afectaron la ladera y el propio sitio CP.

\section{El agua}

El gradiente de precipitación oeste-este disminuye en forma abrupta desde las cumbres andinas (más de $2800 \mathrm{~mm}$ anuales) hasta la estepa, con 300 o menos $\mathrm{mm}$ anuales. En aproximadamente $50 \mathrm{~km}$ se produce el cambio del bosque templado lluvioso a la vegetación xerofítica. De esta forma, en los sitios que acá presentamos las precipitaciones son $1000 \mathrm{~mm}$ en $\mathrm{PL}$, $800 \mathrm{~mm}$ en RA1 y $700 \mathrm{~mm}$ en CP y en todos los casos se concentran en otoño-invierno. La combinación de altas precipitaciones con el hecho de estar concentradas en determinada época del año, junto con las fluctuaciones de la napa freática y la inundación de sitios debido a crecidas del río vinculadas al aumento de su caudal en primavera e inicios del verano, contribuyen a la variación en los niveles de humedad de los sedimentos afectando a la preservación de los materiales arqueológicos.

\section{Métodos y materiales}

Los distintos atributos vinculados a la alteración térmica no intencional se distinguen del tratamiento térmico (Cattáneo et al. 1997-1998; Deal 2012; Frank 2012; Gregg y Grybush 1976). Las primeras son el resultado de la exposición de las rocas a fogones y producen, en general, algún tipo de daño térmico. Éstos se reconocen a partir de rasgos como los hoyuelos, los conos de desprendimiento, las escamaciones, el craquelado o los agrietamientos, las fracturas transversales y la desintegración (Aschero 1975, 1983; Cattáneo et al. 1997-1998; Connor et al. 1989; Gregg y Grybush 1976).

Por otra parte, evaluamos la influencia de la humedad en los materiales a través de la presencia de sustancias adheridas a las superficies de las piezas, distribuidas de manera irregular o cubriéndolas totalmente, como manchas blancas o ligeras películas de sedimento que pueden vincularse con la presencia de flujos de agua de baja energía o con la saturación de agua en los depósitos por períodos prolongados (ver detalles en Burroni et al. 2002, Petraglia y Potts 1994).

\section{La muestra.}

La muestra que analizamos está compuesta por el total de instrumentos, núcleos y desechos de los tres sitios descriptos. En todos los casos tomamos como única unidad de análisis el depósito completo, sin diferenciar niveles, ya que se trata de registros con una baja resolución e integridad. En el caso de $\mathrm{PL}$, la recuperación de diferentes tipos de materiales modernos en distintos sectores del depósito, hasta casi un metro por debajo de la actual superficie, las inversiones en los fechados y remontajes de tiestos separados verticalmente $90 \mathrm{~cm}$. nos indican que estamos en presencia de un palimpsesto (Carballido et al. 2016). Por otra parte, durante la excavación de RA1 identificamos numerosas raíces de arbustos, restos óseos de roedores, cuevas de animales cavadores y una importante actividad de lombrices que afectaron la integridad del depósito. A ello se suma la acción de procesos diagenéticos vinculados con el agua que se registra en gran parte de la evidencia recuperada (Podestá et al. 2007) y la información de los pobladores locales que refieren a frecuentes inundaciones que cubren al sitio. Finalmente, en CP la decisión de unificar los niveles se sostiene en que no se registraron diferencias desde el punto de vista sedimentario entre los depósitos con restos arqueológicos. A esto se suma que hemos registrado la acción conjunta de varios agentes, como el fuego (tanto el de incendios forestales como el de fogones antrópicos actuales), el pisoteo, la acción de raíces y el viento, (Bellelli et al. 2003; Carballido Calatayud 2009; Fernández 2006).

En este trabajo se estudió una muestra de 9489 piezas. En PL son 39 instrumentos, 5 núcleos y 1461 desechos de talla, en RA1 9 instrumentos, 1 núcleo y 563 desechos de talla y en CP 86 instrumentos, 10 núcleos y 7315 desechos de talla.

\section{Resultados}

\section{Daños térmicos}

En los tres sitios hemos registrado una elevada proporción de material lítico alterado térmicamente, aunque en proporciones muy distintas en cada uno. En términos generales el $40 \%$ del total de la muestra analizada exhibe algún tipo de daño producto de la exposición al fuego (Tablas 1 y 2). El sitio que presenta la mayor frecuencia de daños térmicos es CP, con el $45 \%$ de los desechos de talla y el $68 \%$ de los instrumentos y núcleos. En menor medida, los desechos de talla de RA1 con este tipo de 
Tabla 1: Alteraciones térmicas sobre materias primas de instrumentos y núcleos. Referencias: HO: hoyuelos. CO: color. CR: craquelado. TM: alteraciones térmicas múltiples. C/H: color+hoyuelos. H/C: hoyuelos+craquelado. C/C: color+craquelado.

Table 1: Thermal damage and raw material. Artifacts and cores. References: HO: pots; CO: color alteration. CR: cracking. TM: multiple thermal alterations. C/H: color+pots. H/C: pots+cracking. C/C: color+cracking.

\begin{tabular}{|c|c|c|c|c|c|c|c|c|c|c|c|}
\hline & & HO & $\mathrm{CO}$ & CR & TM & $\mathrm{C} / \mathrm{H}$ & $\mathrm{H} / \mathrm{C}$ & $\mathrm{C} / \mathrm{C}$ & $\begin{array}{l}\mathrm{N} \text { con } \\
\text { daños }\end{array}$ & $\begin{array}{c}\text { Sin } \\
\text { daños }\end{array}$ & $\begin{array}{c}\mathrm{N} \\
\text { Total }\end{array}$ \\
\hline \multirow{8}{*}{ 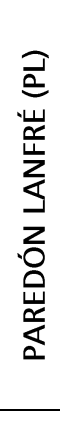 } & sílice & 1 & 4 & & 2 & & & & 7 & 13 & 20 \\
\hline & calcedonia & & 5 & & 1 & & & & 6 & 6 & 12 \\
\hline & toba silicif. & & & & & & & & & 3 & 3 \\
\hline & obsidiana & & & & & & & & & 1 & 1 \\
\hline & andesita & & & & & & & & & 2 & 2 \\
\hline & basalto & & & & & & & & & 4 & 4 \\
\hline & xilópalo & & & & & & & & & 1 & 1 \\
\hline & indif. & & & & 1 & & & & 1 & & 1 \\
\hline & TOTAL PL & 1 & 9 & & 4 & & & & 14 & 30 & 44 \\
\hline \multirow{5}{*}{$\bar{\nwarrow}$} & sílice & & 2 & & 2 & & & 1 & 5 & 1 & 6 \\
\hline & calcedonia & & & & 2 & & & & 2 & & 2 \\
\hline & obsidiana & & & & & & & & & 1 & 1 \\
\hline & basalto & & 1 & & & & & & 1 & & 1 \\
\hline & TOTAL RA1 & & 3 & & 4 & & & 1 & 8 & 2 & 10 \\
\hline \multirow{8}{*}{ 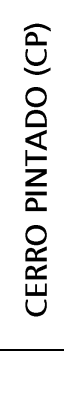 } & limolita & & 1 & & & & & & 1 & & 1 \\
\hline & silice & & 12 & 1 & 3 & & 2 & 2 & 20 & 8 & 48 \\
\hline & calcedonia & & 8 & 3 & 7 & & & 3 & 21 & 10 & 52 \\
\hline & toba silicif. & & 1 & & 2 & & & & 3 & 1 & 7 \\
\hline & obsidiana & & & 1 & 1 & & & & 2 & 6 & 10 \\
\hline & basalto & 1 & 1 & & 1 & & & & 3 & 4 & 10 \\
\hline & indif. & & 3 & & 11 & & & 1 & 15 & & 30 \\
\hline & TOTAL CP & 1 & 26 & 5 & 25 & & 2 & 6 & 65 & 31 & 96 \\
\hline
\end{tabular}

experimentales (Cattáneo et al. 1997-1998, Nami et al. 2000) distintas rocas presentan daños térmicos a diferentes temperaturas, aunque se ha considerado como límite para las rocas con alto contenido de cuarzo los $573^{\circ}$, temperatura a la que este elemento se altera de forma tal que las rocas se vuelven no aptas para la talla (Nami et al. 2000:317). Los resultados que presentamos aquí, en cierto modo corroboran los datos experimentales ya que, por ejemplo, en los desechos de talla de los tres conjuntos es muy alta la incidencia de daños térmicos sobre las rocas silíceas. El sílice y la calcedonia son las materias primas más afectadas: en PL $24 \%$ y el $18,5 \%$, en RA 1 el $34 \%$ y el $20 \%$ y en CP el $39 \%$ y el $36 \%$ respectivamente. Las rocas volcánicas muestran porcentajes menores de daños; por ejemplo en PL la andesita alcanza el

daños alcanzan el $29 \%$ y los instrumentos y núcleos el $80 \%$. Finalmente, en PL el 23\% de los desechos de talla y el $34 \%$ de los instrumentos y núcleos presentan daños térmicos.

En las tablas 1 y 2 también puede observarse la distribución por tipo de daño térmico y por materia prima. En toda la muestra el cambio de color, que en general se asocia al calcinado blanco o gris (Figura 2a y b), es el que predomina, acompañado en algunos casos, por el craquelado y/o por los hoyuelos (Figura 2b y c), combinaciones que se presentan mayoritariamente en CP. Los daños térmicos múltiples (Figura 2a), que combinan tres o más daños, también son muy importantes en los tres conjuntos.

Además, consideramos la distribución de las piezas con estos daños por materia prima ya que según trabajos
$4,6 \%$ y en RA 1 el $13 \%$. Un caso especial lo constituye CP donde esta roca presenta un $40 \%$ de daños mientras que la riolita y el basalto el $55 \%$ y $28 \%$ respectivamente. En este sitio todas las materias primas tanto entre los desechos de talla como entre los instrumentos y núcleos presentan piezas con daños térmicos en proporciones elevadas. Estos valores podrían deberse a la combinación entre la baja tasa de sedimentación, los frecuentes incendios registrados en la ladera del sitio y la quema de raíces de arbustos que han sido señalados como otra fuente significativa de calor (Connor et al. 1989).

Finalmente, analizamos la dispersión espacial de los desechos de talla quemados ya que conforman la gran mayoría de la muestra, con el fin de evaluar su relación con las estructuras de combustión. En el caso de PL, como dijimos, se reconocieron dos estructuras de combustión contiguas, en la base de la excavación. El porcentaje de desechos con daño térmico recuperados 
Tabla 2: Alteraciones térmicas sobre materias primas de desechos. Referencias: HO: hoyuelos. CO: color. CR: craquelado. TM: alteraciones térmicas múltiples. $\mathrm{C} / \mathrm{H}$ : color+hoyuelos. H/C: hoyuelos+craquelado. C/C: color+craquelado.

Table 2: Thermal damage and raw material. Debris. References: HO: pots; CO: color alteration. CR: cracking. TM: multiple thermal alterations. C/H: color+pots. H/C: pots+cracking. C/C: color+cracking.

\begin{tabular}{|c|c|c|c|c|c|c|c|c|c|c|c|}
\hline & & $\mathrm{HO}$ & $\mathrm{CO}$ & CR & TM & $\mathrm{C} / \mathrm{H}$ & $\mathrm{H} / \mathrm{C}$ & $\mathrm{C} / \mathrm{C}$ & $\begin{array}{l}\mathrm{N} \text { con } \\
\text { daños }\end{array}$ & $\begin{array}{c}\text { Sin } \\
\text { daños }\end{array}$ & $\begin{array}{c}\mathbf{N} \\
\text { Total }\end{array}$ \\
\hline \multirow{12}{*}{ 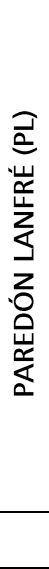 } & sílice & 14 & 61 & 3 & 54 & 5 & & 2 & 139 & 440 & 579 \\
\hline & calcedonia & 1 & 42 & & 26 & 1 & 1 & 2 & 73 & 321 & 394 \\
\hline & obsidiana & 4 & & & & & & & 4 & 29 & 33 \\
\hline & riolita & 3 & & & 1 & & & & 4 & 23 & 27 \\
\hline & andesita & & 4 & & 7 & & & & 11 & 226 & 237 \\
\hline & basalto & & 2 & & & & & & 2 & 60 & 62 \\
\hline & dacita & & & & & & & & & 2 & 2 \\
\hline & cuarzo & & & & & & & & & 1 & 1 \\
\hline & granito & & & & & & & & & 3 & 3 \\
\hline & opalo & & & & & & & & & 2 & 2 \\
\hline & indif. & & 36 & & 66 & 2 & & & & 17 & 121 \\
\hline & TOTAL PL & 22 & 145 & 3 & 154 & 8 & 1 & 4 & 233 & 1124 & 1461 \\
\hline \multirow{9}{*}{ 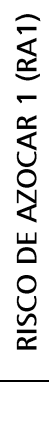 } & sílice & 2 & 58 & & 14 & & & 1 & 75 & 147 & 222 \\
\hline & calcedonia & & 24 & 1 & 4 & & & 1 & 30 & 120 & 150 \\
\hline & obsidiana & & & & & & & & & 31 & 31 \\
\hline & riolita & & 2 & & 1 & & & & 3 & 14 & 17 \\
\hline & andesita & & 7 & & 2 & & & & 9 & 60 & 69 \\
\hline & dacita & & & & & & & & & 4 & 4 \\
\hline & opalo & & & & & & & & & 1 & 1 \\
\hline & indif. & & 23 & & 20 & & & & 43 & 26 & 69 \\
\hline & TOTAL RA1 & 2 & 114 & 1 & 41 & & & 2 & 160 & 403 & 563 \\
\hline \multirow{10}{*}{ 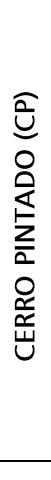 } & sílice & 30 & 519 & 26 & 132 & 98 & 5 & 123 & 933 & 1461 & 2394 \\
\hline & calcedonia & 7 & 533 & 52 & 89 & 34 & 4 & 178 & 897 & 1579 & 2476 \\
\hline & toba silicif. & & 24 & 1 & & & & 5 & 30 & 19 & 49 \\
\hline & obsidiana & 1 & 37 & 1 & 1 & & & 2 & 42 & 188 & 230 \\
\hline & limolita & 1 & 25 & 1 & 3 & & & 2 & 32 & 59 & 91 \\
\hline & arenisca & & 8 & & 2 & 1 & & 1 & 12 & 9 & 21 \\
\hline & basalto & 14 & 190 & 2 & 9 & 20 & & 9 & 244 & 629 & 873 \\
\hline & riolita & & 38 & & 13 & 4 & & 9 & 64 & 53 & 117 \\
\hline & andesita & & 21 & & 6 & & & 2 & 29 & 43 & 72 \\
\hline & indif. & & 282 & & 493 & 53 & 1 & 151 & 980 & 12 & 992 \\
\hline & TOTAL CP & 53 & 1677 & 83 & 748 & 210 & 10 & 482 & 3263 & 4052 & 7315 \\
\hline
\end{tabular}

explicarían como resultado de los fogones actuales, registrados en superficie, ya que los materiales con daño térmico se distribuyen en toda la secuencia estratigráfica sin diferencias significativas.

Acción del agua.

Por otra parte, hemos registrado rasgos que pueden vincularse a la acción del agua sobre los materiales líticos (Tabla 3). Una porción elevada de las piezas de PL (57\%) y de RA1 $(91,7 \%)$ presentan una o ambas caras completamente cubiertas con una película de sedimento adherido (en ocasiones sumada a manchas blancas) dato que nos permite inferir que estuvieron en ambientes húmedos o incluso anegados por tiempos prolongados en los que precipitó sobre las piezas el sedimento local (Figura 2d y f). En CP esta proporción es menor, aunque se encuentra presente en un $42 \%$. Por otra parte, registramos la presencia, en baja frecuencia, de manchas blancas distribuidas de manera heterogénea sobre las caras de algunas piezas que en ninguno de los tres sitios superan el $2 \%$ del conjunto (Figura $2 \mathrm{e}$ ).

\section{Discusión y conclusiones}

El análisis de estos conjuntos muestra una incidencia muy significativa de los daños atribuidos a la acción del fuego. Registramos toda la variedad de

en las estructuras y microsectores adyacentes es 23\%, similar al porcentaje total del sitio. En CP ocurre algo similar, el registro de desechos con daños térmicos en capas y microsectores con fogones alcanza el 40,7\% que es levemente inferior al del sitio. Por último, en RA1 no se identificaron estructuras de combustión por lo que esto no pudo ser evaluado. Estos valores muestran que el descarte en las pocas estructuras de combustión identificadas en los aleros o en los sectores adyacentes no alcanzaría para explicar los elevados porcentajes de materiales termoalterados, como tampoco se daños descripta en la bibliografía, siendo los principales el cambio de color y los combinados, aunque también se presentan hoyuelos y lascas de desprendimiento térmico, agrietamientos y fracturas, lo que hablaría de una exposición prolongada y/o elevadas temperaturas. Teniendo en cuenta la distribución espacial relativamente homogénea de los materiales con daños térmicos y su escasa o nula correlación con la presencia de estructuras de combustión claramente definidas, podríamos argumentar que uno de los principales factores que intervino en esta conformación fueron los incendios 
Tabla 3: Adherencias.

Table 3: Adhered materials.

\begin{tabular}{|l|r|r|r|r|r|r|r|}
\cline { 2 - 7 } \multicolumn{1}{c|}{} & \multicolumn{2}{c|}{ Sedimento } & \multicolumn{2}{c|}{$\begin{array}{c}\text { Sedimento+ } \\
\text { manchas blancas }\end{array}$} & \multicolumn{2}{c|}{ Manchas blancas } & \multirow{2}{*}{$\begin{array}{c}\text { N TOTAL DEL } \\
\text { CONJUNTO }\end{array}$} \\
\cline { 2 - 7 } \multicolumn{1}{c|}{} & \multicolumn{1}{c|}{$\mathrm{N}$} & \multicolumn{1}{c|}{$\mathrm{N}$} & \multicolumn{1}{c|}{$\%$} & $\mathrm{~N}$ & \multicolumn{1}{c|}{$\%$} & 1505 \\
\hline PL & 853 & 56.7 & 7 & 0.46 & 7 & 0.46 & 573 \\
\hline RA1 & 514 & 89.7 & 11 & 2 & 0 & 0 & 7411 \\
\hline CP & 3030 & 41 & 70 & 0.95 & 83 & 1.12 & \\
\hline
\end{tabular}
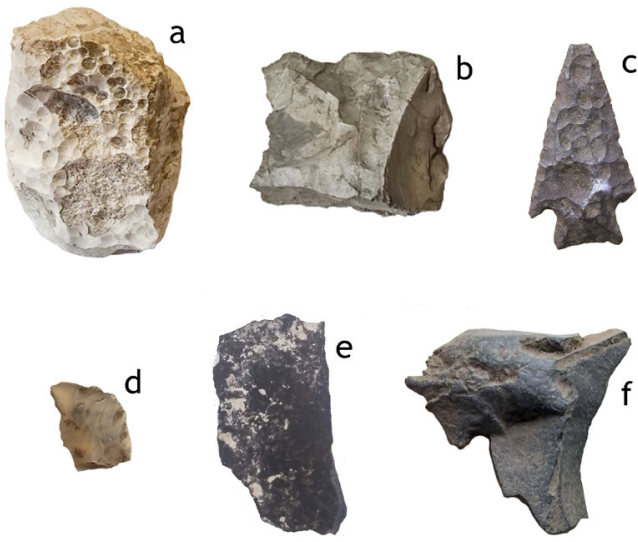

$1 \mathrm{~cm}$

Figura 2: Daños térmicos y adherencias sobre los artefactos líticos. a) Alteraciones térmicas multiples; b) color y craquelado; c) hoyuelos y color; d) y f) sedimento adherido; e) manchas blancas adheridas.

Figure 2: Thermal damage and material adhered on lithic artifacts. a) Multiple thermal damage; b) color alteration and cracking; c) color alteration and pots; d) and f) adhered sediment; e) adhered white spots.

forestales, probablemente sucedidos a lo largo del tiempo. También hemos acercado evidencia sobre la incidencia del fuego en los distintos tipos de materias primas, corroborando lo observado experimentalmente por otros autores, referido a la mayor fragilidad de las rocas con alto contenido de sílice en relación con las volcánicas (Cattáneo et al. 1997-1998, Nami et al. 2000).

Respecto de las adherencias, que pueden relacionarse con la humedad de los ambientes de depositación, RA1 se distingue del resto ya que casi todas las piezas recuperadas presentan esta característica. Su localización en la planicie aluvial y la fuerte incidencia de las inundaciones podrían explicar esta característica. Por su parte, tanto PL como CP (aunque en menor medida) muestran que la humedad, tanto proveniente de las napas freáticas como de las frecuentes precipitaciones, es un agente significativo en la conformación de dichos registros.
Entonces, la particular configuración del registro lítico de esta zona del bosque es el resultado de la combinación de las condiciones microambientales de depositación, las geoformas en las que se emplazan los sitios y la influencia de la dinámica de las perturbaciones naturales (incendios) en el bosque y el régimen de precipitaciones. Es así que registramos algunas tendencias que diferencian a los sitios de acuerdo con su localización. $\mathrm{CP}$, que presenta la más baja tasa de sedimentación y en el que los materiales han estado expuestos más tiempo a recurrentes eventos de fuego, e incluso al uso actual por los visitantes, presenta frecuencias muy elevadas de materiales líticos con todo el abanico de daños térmicos. Por su parte, PL y RA1 con tasas de sedimentación más altas y altamente vegetados, expuestos a la incidencia de los incendios forestales, exhiben porcentajes menores, aunque importantes, de la acción del fuego. Por otra parte, RA1 y PL, ubicados en planicies aluviales, presentan los porcentajes más alto de adherencias, destacándose RA1.

Analizar en detalle la frecuencia y tipos de daños térmicos en los materiales líticos permite evaluar la magnitud de los incendios forestales como formadores del registro a lo largo del tiempo. Además, advierte sobre los efectos que este fenómeno puede ocasionar en materiales más sensibles al calor, que incluso podrían desaparecer o cambiar de estado. Del mismo modo, los depósitos sedimentarios registrados sobre los artefactos líticos también muestran la alta incidencia del otro agente de formación, el agua, que incluso también puede destruir o cambiar el estado de otro tipo de evidencia. Como corolario de este trabajo vemos que el peso de los incendios forestales y de la elevada humedad en los depósitos como agentes de formación del registro lítico en el bosque es muy significativo y probablemente sean aquellos que han modelado los conjuntos que hallamos en este ambiente.

Buenos Aires, 24 de abril de 2019.

\section{Agradecimientos}

Agradecemos especialmente a los pobladores del valle del río Manso, del valle del río Epuyén y de Cholila que durante años nos han ofrecido su hospitalidad y cariño y nos han brindado sus conocimientos y experiencias. Gracias a Ana Forlano por la figura, a Pablo Fernández y Vivian Scheinsohn por los intercambios de ideas que 
finalmente son de todos. La paciencia, la cordialidad y el trato amable a los editores de este volumen merecen nuestro profundo agradecimiento. También estamos en deuda con los dos evaluadores que con sus comentarios ayudaron a que este trabajo fuera más claro y completo. Las instituciones que apoyaron nuestro trabajo son la Agencia Cultura de la Provincia de Río Negro, la Secretaría de Cultura de la Provincia de Chubut y el INAPL. El financiamiento estuvo a cargo del CONICET, la UBA y la ANPCyT.

\section{Referencias citadas.}

Aschero, C. A. (1975). Ensayo para una clasificación morfológica de artefactos líticos aplicada a estudios tipológicos comparativos. Informe presentado al CONICET. Manuscrito inédito.

Aschero, C. A. (1983). Ensayo para una clasificación morfológica de artefactos líticos. Apéndices A y B. Cátedra de Ergología y Tecnología, Facultad de Filosofía y Letras, UBA. Manuscrito inédito.

Bellelli, C., M. Carballido, P. Fernández \& V. Scheinsohn (2003). El pasado entre las hojas. Nueva información arqueológica del noroeste de la provincia de Chubut, Argentina. Werken 4, 25-42.

Bellelli, C., M. Carballido Calatayud, P. M. Fernández \& V. Scheinsohn (2007). Investigaciones arqueológicas en el valle del río Manso Inferior (Pcia. de Río Negro). Actas del XVII Congreso Nacional de Arqueología Argentina. Tras las huellas de la materialidad. Resúmenes Ampliados. Tomo III (pp. 309-314). San Salvador de Jujuy: Facultad de Humanidades y Ciencias Sociales, Universidad Nacional de Jujuy.

Burroni, D, R. E. Donahue \& A. M. Pollard (2002). The surface alteration features of flint artefacts as a record of environmental processes. Journal of Archaeological Science, 29, 1277-1287. DOI:10.1006/jasc.2001.0771

Carballido Calatayud, M. (2009). Evaluación del registro lítico de superficie en la localidad de Cholila (Chubut): un problema nada superficial. En M. Salemme, F. Santiago, M. Álvarez, E. Piana, M. Vázquez y M. E. Mansur (Eds.), Arqueología de Patagonia. Una mirada desde el último confín (315-326). Ushuaia: Editorial Utopías.

Carballido Calatayud, M., P. Fernández, F.X. Pereyra \& A. Forlano (2016). Un árbol para ver el bosque. El uso del bosque en el tramo final del Holoceno desde la perspectiva del sitio Paredón Lanfré (Valle del Manso, Suroeste de Río Negro). Ponencia presentada en el XIX Congreso Nacional de Arqueología Argentina. San Miguel de Tucumán. Manuscrito inédito.

Cattáneo, R., A. Pupio, M. Valente \& A. Varna (1997-
1998). Alteración térmica en dos tipos de rocas silíceas: resultados experimentales y aporte de datos para el análisis arqueológico. Relaciones 22-23, 343-361.

Connor, M.A., K.P. Cannon \& D.C. Carlevato (1989). The mountains burnt: forest fires and site formation processes. North American Archaeologist, 10(4), 293-310.

Deal, K. (2012). Fire effects on flaked stone, ground stone, and other stone artifacts. En K. Ryan, A.T. Jones, C. Koerner \& K. M. Lee (Eds.), Wildland fire in ecosystems. Effects of Fire on Cultural Resources and Archaeology (97-110). Rocky Mountain Research Station: United States Department of Agriculture Forest Service.

Fernández, P.M. (2006). Aprovechamiento de recursos faunísticos en los ambientes de estepa y ecotono bosque-estepa del norte de la Provincia del Chubut. (Tesis de Doctorado inédita). Facultad de Filosofía y Letras, Universidad de Buenos Aires, Buenos Aires.

Fernández, P. M., M. Carballido Calatayud, C. Bellelli \& M. Podestá (2013). Tiempo de cazadores. Cronología de las ocupaciones humanas en el valle del río Manso inferior (Río Negro). En A. F. Zangrando, R. Barberena, A. Gil, G. Neme, M. Giardina, L. Luna, C. Otaola, S. Paulides, L. Salgán y A. Tívoli (Eds.) Tendencias teóricometodológicas y casos de estudio en la arqueología de la Patagonia (167-175). San Rafael-Buenos Aires: Museo de Historia Natural de San Rafael, Sociedad Argentina de Antropología e Instituto Nacional de Antropología y Pensamiento Latinoamericano.

Fuentes Quezada, F. (1994). Qué futuro tienen nuestros bosques: hacia la gestión sustentable del paisaje del centro y sur de Chile. Santiago: Universidad Católica de Chile.

Frank, A. (2012). Tratamiento y daño térmico de artefactos líticos en los componentes tempranos del sitio Casa del Minero 1, Santa Cruz, Argentina. Chungara, Revista de Antropología Chilena, 44 (1), 25-37.

Gregg, M. L. \& R. J. Grybush (1976). Thermally altered siliceous stone from prehistoric contexts: intentional versus unintentional alteration. American Antiquity 41 (2), 189-192.

Giaché. Y. \& M.M. Bianchi (2018). 13,000 years of forest history, in the Río Manso Inferior Valley, Northern Patagonia. Fire-vegetation-humans links. Journal of Archaeological Science: Reports 18, 769-774. https://doi. org/10.1016/j.jasrep.2017.09.001

Iglesias, V., C. Whitlock, M.M. Bianchi, G. Villarosa \& V. Outes (2012). Holocene climate variability and environmental history at the Patagonian forest/steppe ecotone: Lago Mosquito $\left(42.50^{\circ} \mathrm{S}, 71.40^{\circ} \mathrm{W}\right)$ and Laguna 
del Cóndor $\left(42.20^{\circ} \mathrm{S}, 71.17^{\circ} \mathrm{W}\right)$. The Holocene 22, 1297-1307.

Kitzberger, T. \& T. Veblen (1999). Fire-induced changes in northern Patagonian landscape. Landscape Ecology $14,1-15$.

Nami, H.G., R. Cattaneo \& M.A. Pupio (2000). Investigaciones experimentales sobre el tratamiento térmico en algunas materias primas de Pampa y Patagonia. Anales del Instituto de la Patagonia, Serie Cs.Hs. 28, 315-329.

Petraglia, M.D. \& R. Potts (1994). Water flow and the formation of early Pleistocene artifact sites in Olduvai gorge, Tanzania. Journal of Anthropologial Archaeology 13, 228-254.

Podestá, M., Bellelli, C., Fernández, P., Scheinsohn, V., Carballido Calatayud, M., Forlano, A., Marchione, P., Tropea, E., Vasini, A., Alberti, J., Gallo, M. \& Moscovici Vernieri, G. (2007). Arqueología del valle del río Epuyén (El Hoyo, Chubut, Patagonia argentina). En Morello,F. M. Martinic, A. Prieto y C.Bahamonde (Eds.) Arqueología de Fuego-Patagonia. Levantando piedras, desenterrando huesos... y develando arcanos (427-442). Punta Arenas: Ediciones CEQUA. Centro de Estudios del Hombre Austral, Instituto de la Patagonia-Universidad de Magallanes. 